NASA/TM-1998-206613

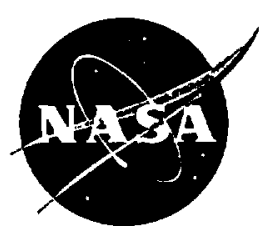

\title{
Calculation of Multistage Turbomachinery Using Steady Characteristic Boundary Conditions
}

Rodrick V. Chima

Lewis Research Center, Cleveland, Ohio

Prepared for the

36th Aerospace Sciences Meeting \& Exhibit

sponsored by the American Institute of Aeronautics and Astronautics

Reno, Nevada, January 12-15, 1998

National Aeronautics and

Space Administration

Lewis Research Center 
Available from

NASA Center for Aerospace Information 800 Elkridge Landing Road

Linthicum Heights, MD 21090-2934

Price Code: $\mathrm{AO3}$
National Technical Information Service 5287 Port Royal Road Springfield, VA 22100 Price Code: $\mathrm{A} 03$ 


\title{
Calculation of Multistage Turbomachinery Using Steady Characteristic Boundary Conditions
}

\author{
Rodrick V. Chima \\ NASA Lewis Research Center \\ Cleveland, Ohio 44135
}

\begin{abstract}
A multiblock Navier-Stokes analysis code for turbomachinery has been modified to allow analysis of multistage turbomachines. A steady averaging-plane approach was used to pass information between blade rows. Characteristic boundary conditions written in terms of perturbations about the mean flow from the neighboring blade row were used to allow close spacing between the blade rows without forcing the flow to be axisymmetric. In this report the multiblock code is described briefly and the characteristic boundary conditions and the averaging-plane implementation are described in detail. Two approaches for averaging the flow properties are also described. A two-dimensional turbine stator case was used to compare the characteristic boundary conditions with standard axisymmetric boundary conditions. Differences were apparent but small in this low-speed case. The two-stage fuel turbine used on the space shuttle main engines was then analyzed using a three-dimensional averaging-plane approach. Computed surface pressure distributions on the stator blades and endwalls and computed distributions of blade surface heat transfer coefficient on three blades showed very good agreement with experimental data from two tests.
\end{abstract}

\section{Introduction}

Computational methods for analyzing steady flows in isolated turbomachinery blade rows are now highly developed and commonly used for turbomachinery design. Except for some fans and pumps, however, few turbomachines operate as isolated blade rows. Most turbomachines include at least a stator to add or remove

\footnotetext{
† Aerospace Engineer, Associate Fellow AIAA

Copyright $(1998$ by the American Institute of Aeronautics and Astronautics, Inc. No copyright is asserted in the United States under Title 17, U.S. Code. The U.S. Government has a royalty-free license to exercise all rights under the copyright claimed herein for government purposes. All other rights are reserved by the copyright owner.
}

swirl, and often include many stages to do more work than could be accomplished with a single blade row.

Several methods exist for analyzing flows in multistage turbomachinery. They include the following: 1 . successive analysis of isolated blade rows, 2 . averagingplane methods, 3. the average-passage method, and 4. full unsteady methods. Each method has advantages but also introduces modeling issues, as discussed below.

\section{Successive Analysis of Isolated Blade Rows}

Given an analysis code for an isolated blade row, it is tempting to simulate multistage turbomachinery by analyzing successive blade rows from inlet to exit, using average flow properties from the exit of one blade row as inlet boundary conditions for the next. This method is simple, but it introduces many modeling issues. First, since blade rows are often closely spaced, it is unclear how far to extend the computational grid for each blade row, and whether it is reasonable to overlap grids. Second, many numerical boundary conditions are not wellbehaved when applied too close to a blade. Third, average flow properties are not well-defined [1]. Since flow properties are related nonlinearly, it is impossible to define an average state that maintains all the original properties of the three-dimensional flow. Fourth, for subsonic flow, the inlet velocity profile and massflow develop as part of the solution. Although it may be possible to match the overall massflow by iterating on the imposed back pressure, it is generally not possible to match the spanwise distributions of properties between the blade rows. Finally, the method ignores physical processes such as wake mixing and migration, acoustic interaction, and other unsteady effects that may be important in real turbomachinery.

Many researchers have used successive analysis of isolated blade rows to model multistage turbomachines. Boyle and Giel used this method to analyze the fuel turbine of the space shuttle main engine (SSME) [2]. This turbine was also analyzed in the present work.

\section{Averaging-Plane Methods}

Averaging-plane methods solve all blade rows simultaneously, exchanging spanwise distributions of 
averaged flow quantities at a common grid interface between the blade rows. These methods have the advantage of maintaining spanwise consistency between blade rows, but share the modeling issues of boundary condition implementation, averaging techniques, and missing physics with the successive analysis method. Since averaging-plane methods often use mixed-out averages, they are commonly referred to as mixing-plane methods. The current work is independent of the averaging technique, so the term averaging-plane will be used.

Averaging-plane methods were introduced simultaneously by Denton [3] and Dawes [4], and have been used by many other researchers [5 - 9]. In spite of the possibility of missing physics in these analyses, many have shown excellent agreement with experimental data.

\section{Average-Passage Method}

The average-passage method was developed by Adamczyk, et. al [10 - 12] as a rigorous means of modeling unsteady blade row interaction using a steady analysis. The method splits the flow quantities into a steady component, an unsteady deterministic (periodic) component, and an unsteady random (turbulent) component. The flow equations are integrated in time using procedures analogous to Reynolds averaging to produce the average-passage equations. The integration process produces the usual Reynolds stress terms, as well as correlations for deterministic stress terms that must be modeled. The average-passage method has the advantage of a rigorous foundation for modeling unsteady blade-row interaction, although little data is available for modeling the deterministic stresses. The method requires that the computational grids for each blade overlap at least one neighboring blade row on each side, adding to programming complexity and computational overhead.

The average-passage method has been used for numerous applications by Adamczyk, et. al [10 - 12], and by Rhie et al. [13] and LaJambre et al. [14] for turbine design, but because of its complexity it has not been widely used by others. Recently Hall has described an algebraic method for adding some of the averagepassage terms to an averaging-plane analysis $[6,7]$.

\section{Full Unsteady Methods}

Full unsteady methods, pioneered by Rai [15], involve direct solution of unsteady rotor-stator interaction. These methods presumably avoid all modeling questions except for turbulence, and are often used to validate other steady models $[1,6,9,16]$. Since turbomachine blade rows usually have different numbers of blades in each row to avoid resonances, full unsteady methods often modify the blade spacing to produce small integral blade ratios. Full unsteady methods are very expensive computationally, and still require averaging at the end to produce useful results.

\section{Boundary Conditions}

For each of the analysis methods mentioned above, boundary conditions must be specified at the inlet and exit of the computational domain. In addition, for averaging-plane methods, average flow properties must be transferred between the blade rows at grid interfaces. It is common practice to force the flow to be axisymmetric at these boundaries. Although axisymmetric boundary conditions are simple to apply and tend to be numerically robust, they can reflect outgoing waves and thereby hinder convergence and contaminate the interior solution. Axisymmetric boundary conditions can be particularly bad at the inlet of transonic compressors, at the exit of transonic turbines, and between closely-spaced blade rows.

In [17] Giles presented a unified theory for the construction of non-reflecting boundary conditions for the Euler equations. The boundary conditions are based on the linearized Euler equations written in terms of perturbations of primitive variables about some mean flow. Wave-like solutions are substituted into the flow equations, and the solution is circumferentially decomposed into Fourier modes. The zeroth mode corresponds to the mean flow and is treated according to one-dimensional characteristic theory. This allows average changes in incoming characteristic variables to be specified at the boundaries. Reference [17] also describes higher-order two-dimensional boundary conditions, but these were not used in the present work.

Giles demonstrated that his boundary conditions allowed inlet and exit boundaries to be placed very close to turbine blades with no loss of accuracy [17]. Saxer and Giles applied these boundary conditions to an inviscid, three-dimensional solution for a transonic turbine stage [9]. They demonstrated good agreement in blade pressures between a full unsteady solution and an averaging-plane solution. Arnone applied Giles' boundary conditions to a quasi-three-dimensional viscous simulation of a transonic compressor stage [16]. He compared a full unsteady solution with an averaging-plane solution and showed close agreement in predicted pressure ratios and efficiencies between the two.

\section{Present Work}

In the present work an improved averaging-plane method for three-dimensional viscous flows in turbomachinery was developed. The averaging-plane method gives steady solutions of multistage turbomachinery with consistent spanwise profiles between the blade 
rows, but ignores unsteady effects that may be important in real turbomachines. The analysis was based on the SWIFT multi-block code developed by the author [18], which is described here briefly.

Giles' characteristic boundary were used at the averaging planes. The boundary conditions were written in terms of perturbations about the average flow from the neighboring blade row, providing a rational way of coupling the solutions. They allow close spacing between blade rows without forcing the flow to be axisymmetric. The boundary conditions and averaging techniques are described in detail.

Computations were made of the two-stage fuel turbine from the space shuttle main engine. A computational grid with seven blocks and about 1.09 million grid points was used. Comparisons were made with experimental pressure distributions on the stators and endwalls, and with experimental heat transfer distributions on three of the blades.

\section{SWIFT Code}

The SWIFT turbomachinery analysis code is a multiblock version of the single-block RVC3D code described in [19] and [20]. The SWIFT code solves the Navier-Stokes equations on body-fitted grids using an explicit finite-difference scheme. It includes viscous terms in the blade-to-blade and hub-to-tip directions, but neglects them in the streamwise direction using the thinlayer approximation. The Baldwin-Lomax and CebeciSmith turbulence models [21] are available. The code has limited multiblock capability intended solely for turbomachinery problems. Only $\mathrm{C}$-grids for blades, $\mathrm{O}$ grids for hub and tip clearances, $\mathrm{H}$-grids for inlets, and patched $\mathrm{C}$-grids for multistage calculations are currently supported.

An explicit, four-stage Runge-Kutta scheme [22] was used to solve the flow equations. Conservative fourth-difference artificial dissipation terms were added to control point decoupling. (Second-difference terms were not needed for the subsonic flow considered here.) Eigenvalue scaling [23] was used to scale the artificial dissipation directionally on the highly stretched grids. The artificial dissipation was also reduced linearly with grid index near solid surfaces (typically by a factor of 0.05 at the wall) to minimize effects on wall heat transfer. Artificial and physical dissipation terms were computed at the first and second stages to improve numerical smoothing properties. The Cebeci-Smith turbulence model was used, with all boundary layers assumed to be fully turbulent.
To accelerate convergence to a steady state, the calculations were run at a Courant number of 5.6 using a spatially-varying time step and implicit residual smoothing. Eigenvalue scaling was used to minimize the implicit smoothing coefficients at each point in each direction. Preconditioning [24] was also used to improve the convergence rate, since most of the flow in the problem considered here was at relatively low Mach numbers $(0.15$ to 0.45 .)

\section{Characteristic Boundary Conditions}

The general form of the non-reflecting one-dimensional unsteady boundary conditions developed by Giles [17] was used here. The boundary conditions were developed in Cartesian coordinates, but can be applied immediately to cylindrical coordinates if the source term in the radial momentum equation is ignored. The boundary conditions use the following characteristic variables:

$$
\left[\begin{array}{l}
C_{1} \\
C_{2} \\
C_{3} \\
C_{4} \\
C_{5}
\end{array}\right]=\left[\begin{array}{ccccc}
-\bar{c}^{2} & 0 & 0 & 0 & 1 \\
0 & \bar{\rho} \bar{c} & 0 & 0 & 1 \\
0 & 0 & \bar{\rho} \bar{c} & 0 & 0 \\
0 & 0 & 0 & \bar{\rho} \bar{c} & 0 \\
0 & -\bar{\rho} \bar{c} & 0 & 0 & 1
\end{array}\right]\left[\begin{array}{c}
\rho-\bar{\rho} \\
v_{x}-\overline{v_{x}} \\
v_{\theta}-\bar{v}_{\theta} \\
v_{r}-\bar{v}_{r} \\
p-\bar{p}
\end{array}\right]
$$

Equation (1) can be inverted to give:

$$
\left[\begin{array}{c}
\rho-\bar{\rho} \\
v_{x}-\bar{v}_{x} \\
v_{\theta}-\bar{v}_{\theta} \\
v_{r}-\bar{v}_{r} \\
p-\bar{p}
\end{array}\right]=\left[\begin{array}{ccccc}
-1 / \bar{c}^{2} & 1 /\left(2 \bar{c}^{2}\right) & 0 & 0 & 1 /\left(2 \bar{c}^{2}\right) \\
0 & 1 /(2 \bar{\rho} \bar{c}) & 0 & 0 & -1 /(2 \bar{\rho} \bar{c}) \\
0 & 0 & 1 /(\bar{\rho} \bar{c}) & 0 & 0 \\
0 & 0 & 0 & 1 /(\bar{\rho} \bar{c}) & 0 \\
0 & 1 / 2 & 0 & 0 & 1 / 2
\end{array}\right]\left[\begin{array}{l}
C_{1} \\
C_{2} \\
C_{3} \\
C_{4} \\
C_{5}
\end{array}\right]
$$

In equations (1) and (2) $C_{i}$ are characteristic variables corresponding to an entropy wave, a downstreamrunning pressure wave, two vorticity waves, and an upstream-running pressure wave. Here also $\rho$ is the density, $p$ is the pressure, $c$ is the speed of sound, and $v_{x} v_{\theta}$, and $v_{r}$ are velocity components. Overbars refer to average conditions to be defined later, and the coefficient matrices are evaluated at those average conditions.

\section{Inlet Boundary Condition}

For subsonic flow at an inlet boundary, the four incoming characteristics $C_{1}$ through $C_{4}=0$ and the out- 
going characteristic $C_{5}$ is extrapolated from the interior. Substituting $C_{1}$ through $C_{4}=0$ and $C_{5}=C_{5 e x}$ into equation (2) gives:

$$
\begin{aligned}
\rho & =\bar{\rho}+C_{5 e x} /\left(2 \bar{c}^{2}\right) \\
v_{x} & =\bar{v}_{x}-C_{5 e x} /(2 \bar{\rho} \bar{c}) \\
v_{\theta} & =\bar{v}_{\theta} \\
v_{r} & =\bar{v}_{r} \\
p & =\bar{p}+C_{5 e x} / 2
\end{aligned}
$$

where the subscript ( ) ex implies extrapolation from the interior. Equations (3) show that at the inlet $\rho, v_{x}$, and $p$ are modified by the upstream-running characteristic $C_{5}$, while $\overline{v_{\theta}}$ and $\bar{v}_{r}$ convect downstream. A solution of the $\theta$-momentum equation is that $r v_{\theta}$ is constant along streamlines, and it may be desirable to modify the boundary conditions to give this result. For supersonic inflow $C_{5}=0$ and all boundary variables are equal to their specified average values.

\section{Exit Boundary Condition}

For subsonic flow at an exit boundary the incoming characteristic $C_{5}=0$ and four outgoing characteristics $C_{1}$ through $C_{4}$ are extrapolated from the interior. Substituting values for $C_{i}$ into equation (2) gives:

$$
\begin{aligned}
\rho & =\bar{\rho}+\left[-C_{1 e x}+\left(C_{2 e x}+C_{5}\right) / 2\right] / \bar{c}^{2} \\
v_{x} & =\bar{v}_{x}+\left(C_{2 e x}-C_{5}\right) /(2 \bar{\rho} \bar{c}) \\
v_{\theta} & =v_{\theta e x} \\
v_{r} & =v_{r e x} \\
p & =\bar{p}+\left(C_{2 e x}+C_{5}\right) / 2
\end{aligned}
$$

For subsonic outflow $C_{5}=0$ and $\rho, v_{x}$, and $p$ are modified by the downstream-running characteristics $C_{1}$ and $C_{2}$ while $v_{\theta}$ and $v_{r}$ convect downstream. For supersonic outflow $C_{5}=C_{5 e x}$, and equations (4) reduce algebraically to extrapolation of all primitive variables downstream.

A particularly simple exit boundary condition can be devised by extrapolating four primitive variables $\rho, v_{x}, v_{\theta}, v_{r}$ to the exit (conservation variables based on Cartesian velocity components work equally well). Then substituting $C_{2 e x}=\bar{\rho} \bar{c}\left(v_{x, e x}-\bar{v}_{x}\right)+\left(p_{e x}-\bar{p}\right)$ and $C_{5}=0$ into the last equation in (4) gives:

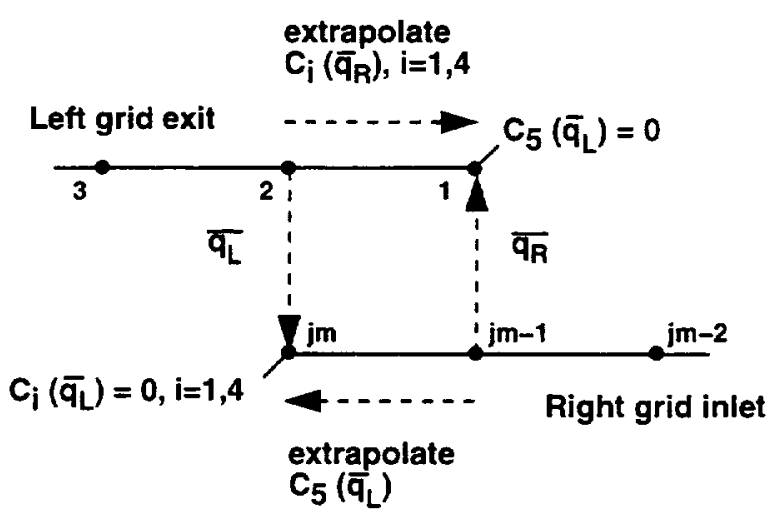

Figure 1 - Implementation of characteristic boundary condition at a blade row interface

$$
p=\frac{1}{2}\left[\bar{p}+p_{e x}+\bar{\rho} \bar{c}\left(v_{x, e x}-\bar{v}_{x}\right)\right]
$$

Equation (5) works well for inviscid flows, including cases with oblique shocks crossing the exit boundary. It was used for the three-dimensional multistage turbine results shown later. After those results were computed it was discovered that equation (5) gives small pressure perturbations $p-\bar{p}$ proportional to velocity perturbations $v_{x}-\bar{v}_{x}$ wherever viscous wakes cross the exit boundary. To reduce these pressure perturbations, equation (5) can be modified by replacing the convective speed $\bar{c}$ with $\left|\vec{v}_{x}\right|$, i.e.,

$$
p=\frac{1}{2}\left[\bar{p}+p_{e x}+\bar{\rho}\left|\bar{v}_{x}\right|\left(v_{x, e x}-\bar{v}_{x}\right)\right]
$$

Two-dimensional computations using equations (5) and (6) are compared later. For three-dimensional turbomachinery calculations equations (5) or (6) can be solved at each spanwise location, with $\bar{p}$ found by solving an average radial equilibrium equation.

\section{Interface Boundary Condition}

For the node-centered finite-difference scheme used in the SWIFT code, computational grids were overlapped by one cell at the interface between two blade rows. This is shown schematically in figure 1 where the two grids have been displaced vertically for clarity. After updating the interior solution on a grid, the solution next to the boundary was integrated circumferentially at each spanwise location as described below. The average flow vector $\bar{q}$ was then stored for use in the boundary conditions on the neighboring grid. On the neighboring grid the average Mach number was checked to determine whether the flow was supersonic, and the 
characteristic boundary conditions (3) or (4) were applied as appropriate. For general (non-axial) turbomachinery problems, (3) and (4) can be modified by replacing the cylindrical velocity components $v_{x}$ and $v_{r}$ with rotated components $v_{s}$ and $v_{n}$, evaluated along streamwise and spanwise grid lines

Although this interface boundary condition does not guarantee conservation between blade rows, experience has shown that it conserves mass and energy between blade rows about as well as the finite difference scheme conserves these properties through the blade rows. Furthermore, the degree of conservation depends on the technique used to average the flow properties at the interface.

\section{Averaging Techniques}

The characteristic boundary conditions described above require average flow properties at the boundaries. In general, any two independent thermodynamic properties and any three independent kinematic properties may be integrated to define some average fluid state. The integrated properties may be chosen to represent certain desirable characteristics of the original flow such as conservation of mass, momentum, and energy. Since flow properties are related nonlinearly, the average properties may not satisfy other characteristics of the original system; that is, information is lost through the averaging process. It thus becomes necessary to decide what information must be retained, and to devise averaging schemes accordingly.

Many averaging techniques have been proposed for use with averaging-plane methods [3-9], and reference [1] contains information on averaging techniques in general. Two averaging techniques were used in the current work, a mixed-out average and a kinetic energy average.

\section{Mixed-Out Average}

Saxer and Giles used a stream-thrust flux-average (also known as a mixed-out average) to conserve mass, momentum, and energy [9]. A similar averaging technique was used by Denton in [3]. The mixed-out average can be derived formally by integrating the two-dimensional Euler equations in the $y$-direction. If the flow is periodic in $y$, the integral of the $y$-direction fluxes is zero. The resulting equation shows that the average $x$ direction flux terms are constant with $x$, i.e., the average properties represent the mixed-out flow far downstream.

When a mixed-out average is used at an exit boundary at which the static pressure has been specified, the average pressure will be less than or equal to the speci- fied pressure. The difference corresponds to the pressure drop required to overcome mixing losses that would occur downstream. The average total pressure includes those mixing losses. Thus, when a mixed-out average is used with an averaging-plane analysis, mixing losses may be introduced prematurely ahead of a blade row.

A mixed-out average can be applied in a generalized cylindrical coordinate system by equating the integrated fluxes to fluxes constructed from the average properties. If the $\eta$-coordinate is assumed to coincide with the $\theta$-direction, then

$$
\left[\begin{array}{l}
I_{1} \\
I_{2} \\
I_{3} \\
I_{4} \\
I_{5}
\end{array}\right]=\int J^{-1}\left[\begin{array}{l}
\rho U \\
\rho v_{x} U+\varepsilon_{x} p \\
\rho v_{\theta} U+\left(\varepsilon_{\theta} / r\right) p \\
\rho v_{r} U+\varepsilon_{r} p \\
e+p U
\end{array}\right] d \eta=\left[\begin{array}{l}
\bar{\rho} \bar{U} \\
\bar{\rho} \overline{v_{x}} \bar{U}+\overline{\varepsilon_{x}} \bar{p} \\
\bar{\rho} \overline{v_{\theta}} \bar{U}+\overline{\left(\varepsilon_{\theta} / r\right) \bar{p}} \\
\bar{\rho} \overline{v_{r}} \bar{U}+\bar{\varepsilon}_{r} \bar{p} \\
(\bar{e}+\bar{p}) \bar{U}
\end{array}\right](7)
$$

where the cylindrical metrics and velocity components can be found from the Cartesian components (used in the SWIFT code) using:

$$
\begin{array}{ll}
U & =\varepsilon_{x} v_{x}+\left(\varepsilon_{\theta} / r\right) v_{\theta}+\varepsilon_{r} v_{r} \\
\varepsilon_{r} & =\left(\varepsilon_{z} z+\varepsilon_{y} y\right) / r \quad \varepsilon_{\theta} / r=\left(\varepsilon_{z} y-\varepsilon_{y} z\right) / r \\
v_{r} & =(v y+w z) / r \\
r & =\sqrt{y^{2}+z^{2}}
\end{array}
$$

Equation (7) gives a quadratic equation for $\bar{p}$. The solution is

$$
\begin{aligned}
& \bar{p}=\frac{1}{\gamma+1}\left[a / b \pm \sqrt{(a / b)^{2}+\left(\gamma^{2}-1\right)\left(c-2 I_{1} I_{5}\right) / b}\right] \\
& a=\bar{\varepsilon}_{x} I_{2}+\overline{\left(\varepsilon_{\theta} / r\right)} I_{3}+\bar{\varepsilon}_{r} I_{4} \\
& b=\bar{\varepsilon}_{x}^{2}+\overline{\left(\varepsilon_{\theta} / r\right)^{2}}+\bar{\varepsilon}_{r}^{2} \\
& c=I_{2}^{2}+I_{3}^{2}+I_{4}^{2}
\end{aligned}
$$

The positive root is used for axially-subsonic flow. The other average properties follow immediately from

$$
\begin{aligned}
\bar{v}_{x} & =\left(I_{2}-\bar{\varepsilon}_{x} \bar{p}\right) / I_{1} \\
\bar{v}_{\theta} & =\left(I_{3}-\overline{\left(\varepsilon_{\theta} / r\right.} \bar{p}\right) / I_{1} \\
\bar{v}_{r} & =\left(I_{4}-\bar{\varepsilon}_{r} \bar{p}\right) / I_{1} \\
\bar{\rho} & =I_{1} / \bar{U}
\end{aligned}
$$

\section{Kinetic Energy Average}

While the mixed-out average formally represents a uniform flow far downstream, the kinetic energy average 
is meant to represent the local state of the flow. It conserves mass and is designed to conserve total enthalpy by individually conserving static enthalpy and the square of each of the velocity components. As a result, the static pressure derived from the kinetic energy average represents an average local pressure, and the total pressure ignores mixing losses that may occur downstream.

Individual velocity components are mass-averaged to give the correct signs and relative magnitudes of the average velocities. An additional mass-average of $v^{2}$ is used to rescale average velocity components such that $\bar{v}_{x}^{2}+\bar{v}_{\theta}^{2}+\bar{v}_{r}^{2}=\bar{v}^{2}$. The integrated properties are given by:

$$
\left[\begin{array}{c}
I_{1} \\
I_{2} \\
I_{3} \\
I_{4} \\
I_{5} \\
I_{6}
\end{array}\right]=\int J^{-1}\left[\begin{array}{l}
\rho U \\
\rho v_{x} U \\
\rho v_{\theta} U \\
\rho v_{r} U \\
\rho U h \\
\rho U V^{2}
\end{array}\right] d \eta
$$

The average properties are given by

$$
\begin{aligned}
& \bar{\rho}=I_{1} / \bar{U} \quad \bar{h}=I_{5} / I_{1} \\
& r=\sqrt{\frac{I_{6}}{I_{1}\left(I_{2}^{2}+I_{3}^{2}+I_{4}^{2}\right)}}=\frac{1}{I_{1}} \sqrt{\frac{\overline{v_{x}^{2}} \bar{v}^{2}+\bar{v}_{\theta}^{2}+\bar{v}_{r}^{2}}{v_{\theta}}=r I_{3} \quad \overline{v_{r}}=r I_{4}}
\end{aligned}
$$

\section{Results}

\section{Space Shuttle Main Engine Fuel Turbine}

Each engine on the space shuttle uses two turbopumps to pump the fuel and oxidizer from the main tank to the combustion chamber. The high-pressure fuel turbopump uses a two-stage axial flow turbine to drive the pump. The turbine blades are cooled by conduction to liquid hydrogen fuel circulated in the disk cavity. The high-pressure fuel turbine (HPFT) was tested experimentally by Hudson, et al. [26] at NASA Marshall Space Flight Center in a cold-flow test. Surface pressures on the stators and endwalls and overall performance parameters were measured in that test. The HPFT was also tested experimentally by Dunn, et al. [25] at Calspan in a short-duration shock tube. Blade surface heat transfer and unsteady pressures were measured in that test.
Computations have been made of flow through the HPFT at the operating conditions tested by Dunn, et al. (referenced by Dunn as run number 12.) The computations are described below, and comparisons are made with the pressure measurements of Hudson, et al. and the heat transfer measurements of Dunn, et al.

\section{Computational Grid}

Grids were generated for each blade separately using the TCGRID turbomachinery grid code, which is described briefly in [20]. The code generated C-type blade-to-blade grids at a few spanwise locations using an elliptic grid generator. The $\mathrm{C}$-grids were then reclustered spanwise using a hyperbolic tangent clustering function. An H-grid was generated upstream of the first stator using transfinite interpolation. O-grids were generated algebraically in the tip clearance region above the two rotors. Grid generation took about one minute per blade row on an SGI workstation with an R4000 processor. Individual grids for each blade were then combined with utility code such that each grid overlapped its neighbor by one cell.

A three-dimensional view of the grid is shown in figure 2. The figure is slightly larger than the actual turbine. The $\mathrm{O}$-grids above the rotor tips can be seen. A meridional projection of the grid is shown in figure 3. For clearance during assembly the trailing edge of stator 1 is cut back over roughly one-third of the span. The cut-back length varies around the wheel, so a nominal length was used here. There is also a step increase in the annulus area between the stages. The precise geometry of the step was unknown, so it was spline-fit arbitrarily between the known radii. Grid sizes are given in table 1 . The nominal initial grid spacings in turbulent wall units were $y^{+}=2.5$ on the blades, $y^{+}=3.5$ on the endwalls, and $y^{+}=7.0$ on the rotor tips.

\section{Effects of Boundary Conditions}

The effects of the characteristic boundary conditions were investigated using two-dimensional calculations of the mid-span section of the first stator. The grid was extracted directly from the multiblock grid described earlier. The exit boundary was located about 0.13 chord lengths downstream axially. Calculations were made using the quasi-three dimensional analysis code described in [1].

Three exit boundary conditions were investigated, and the resulting pressure contours are shown in figure 4 . The contour increment is $\Delta p / p_{0 \text { in }}=0.001$. The solution on the left used a constant-pressure exit boundary condition, as commonly used in averaging-plane analyses. The pressure field near the exit is distorted in com- 


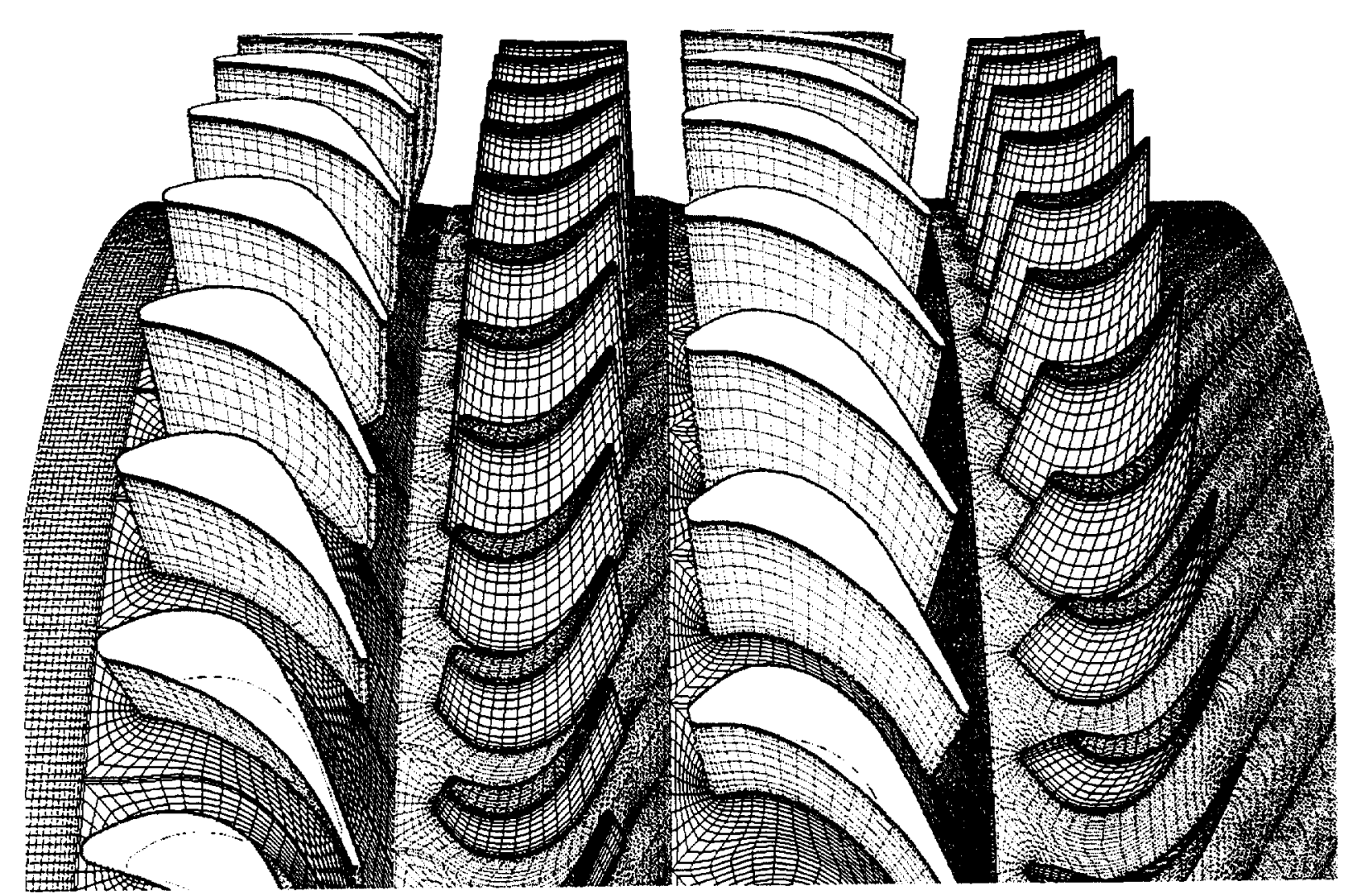

Figure 2 - Multiblock grid for the space shuttle main engine fuel turbine

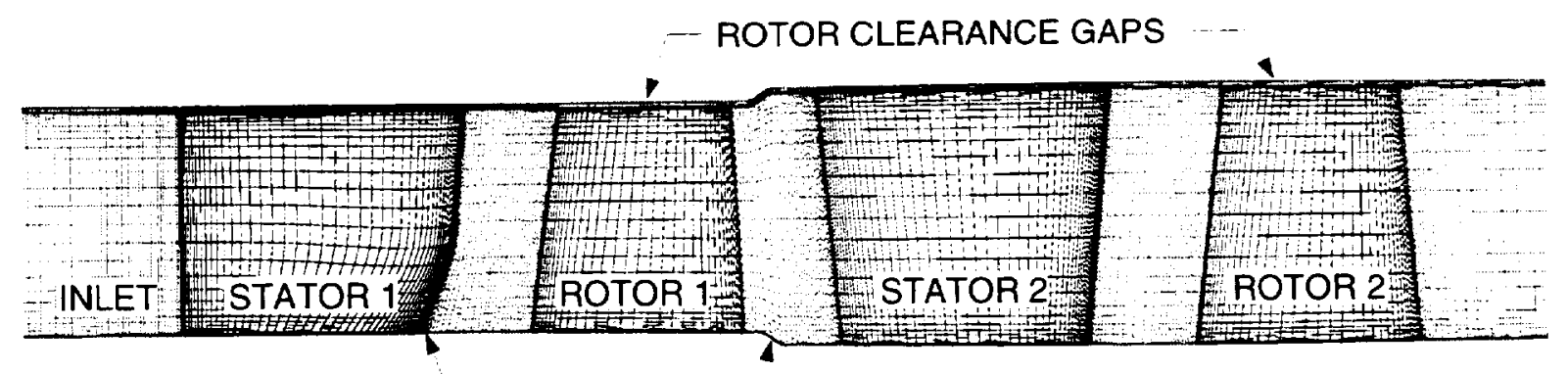
$\begin{array}{ll}\text { STATOR } & \text { STEPPED } \\ \text { CUT-BACK } & \text { ANNULUS }\end{array}$

Figure 3-Meridional view of the computational grid

\begin{tabular}{|l|c|c|c|c|r|}
\hline Grid & \# blades & imax & jmax & kmax & Total \\
\hline inlet & & 17 & 17 & 57 & 16,473 \\
\hline stator 1 & 41 & 127 & 37 & 57 & 267,843 \\
\hline rotor 1 & 63 & 127 & 33 & 57 & 238,887 \\
\hline rotor 1 tip & & 95 & 13 & 13 & 16,055 \\
\hline stator 2 & 39 & 127 & 37 & 57 & 267,843 \\
\hline rotor 2 & 59 & 141 & 33 & 57 & 265,221 \\
\hline rotor 2 tip & & 101 & 13 & 13 & 17,069 \\
\hline Total & & & & & $1,089,391$ \\
\hline
\end{tabular}

Table 1 - Computational grid sizes for SSME fuel turbine. 


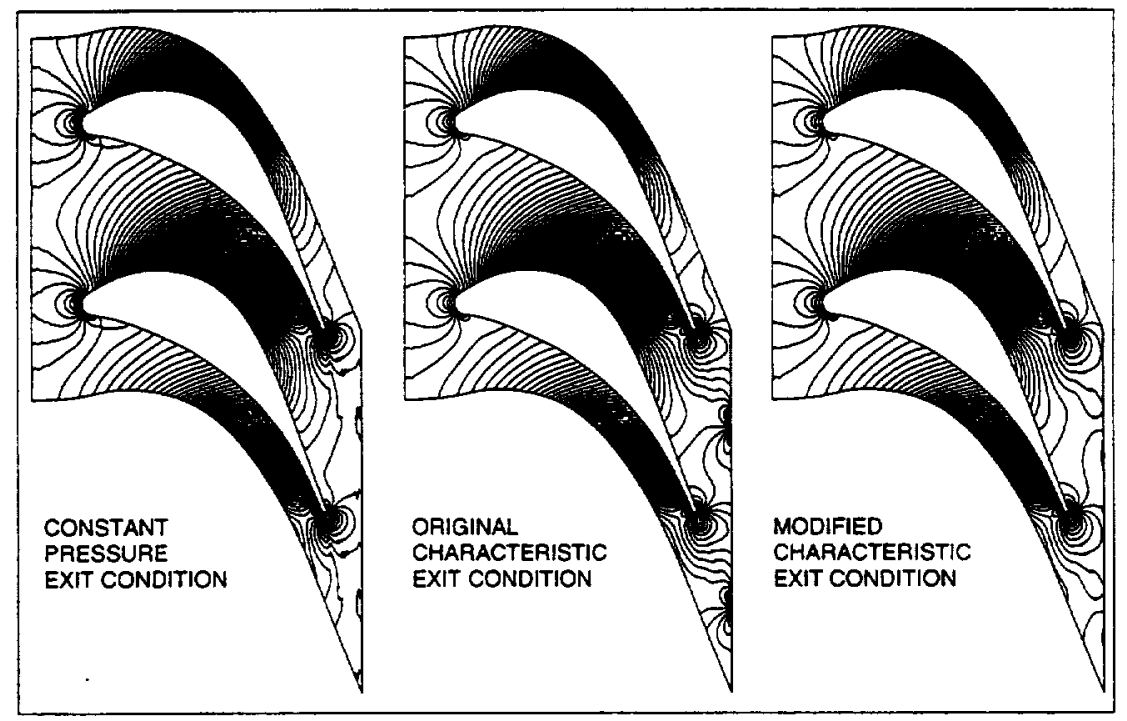

Figure 4 - Comparison of pressure contours for stator 1 computed with three exit boundary conditions

parison to the other solutions in the figure. The solution in the center used the original characteristic exit boundary condition given by equation (5). The pressure field near the exit is much smoother than in the figure on the left, except where the wake (not obvious in the pressure field) crosses the exit boundary. Here pressure perturbations are produced in proportion to the local velocity perturbations. The solution on the right used the modified characteristic exit boundary condition given by equation (6). The pressure field near the exit is smooth and the contours cross the boundary cleanly.

The three solutions have identical average static pressures $\bar{p} / p_{0}=0.86$ at the exit. The surface pressure distribution resulting from the constant-pressure boundary condition is slightly different than the other two solutions on the uncovered part of the suction surface, but the differences are small in this low-speed flow. In transonic cases the differences can be dramatic, as shown by Saxer and Giles [9]. The three solutions had virtually identical convergence behaviors even though the characteristic boundary conditions were designed to transmit outgoing waves and thereby enhance convergence to a steady state.

\section{Multistage Turbine Results}

The multistage turbine was analyzed using the SWIFT code. Boundary conditions were specified to simulate the low-Reynolds number test recorded as run number 12 in reference [25]. At the inlet boundary the total temperature was set to a constant and a total pressure profile was set to produce turbulent boundary layers that were eight percent span thick at the hub and tip. The upstream-running Riemann-invariant was extrapolated from the interior to the inlet, and the primitive variables were calculated as described in [20]. At the exit, the hub static pressure ratio was set to 0.65 to match experimental measurements given in [26], simple radial equilibrium was solved for the mean pressure distribution, and equation (5) was used to calculate the circumferential pressure variation. With this exit pressure distribution the computed flow rate was $2.644 \mathrm{~kg} / \mathrm{sec}(5.83 \mathrm{lb} / \mathrm{sec})$, which was in perfect agreement with the flow rate measured experimentally. At the walls, no-slip boundary conditions were used and the normal pressure gradient was set to zero. The wall/gas temperature ratio was set to 0.7 to approximate the nominal experimental conditions. The characteristic boundary conditions described above were used at the averaging planes.

The calculations were run on the Cray C90 computer at NASA Ames Research Center. They were run 2500 iterations, with a minor change in parameters after 1000 iterations. The convergence history is shown in figure 5 . The calculations required about 25 million words of storage and six hours of CPU time. An initial solution was made using the kinetic energy average at the averaging planes. A second solution was run by restarting from the kinetic energy average solution and running 300 iterations using the mixed-out average.

Figure 6 shows the percent error in mass flow $100 \times\left(\dot{m} / \dot{m}_{i n}-1\right)$ at each computational boundary through the machine. Note that two values are shown at each averaging plane, one corresponding to the upstream exit value, and one corresponding to the down- 


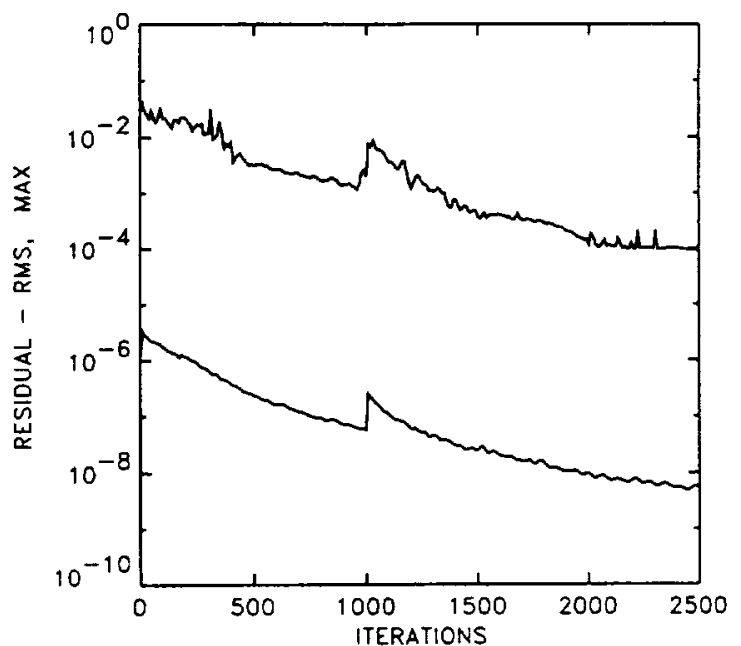

Figure 5 - Residual history for SSME turbine calculation

stream inlet value. No data is shown within the blade row - the lines serve only to connect related points. The dashed line shows the solution using the kinetic energy average. The overall error is less than one percent, but there is a significant jump at each averaging plane. Although the averaging scheme conserves mass, the characteristic boundary conditions allow the solution to vary around the specified averages and the result is not perfectly conservative. The fact that the mass flow increases at each averaging plane appears to be coincidental since other cases have shown decreases at the averaging planes. The solid line shows the solution using the mixed-out average. Here the overall error is less than 0.1 percent and there are practically no errors at the averaging planes. Although the mixed-out average gave better mass conservation than the kinetic energy average, no other obvious differences between the two solutions were found. Other cases at higher speeds or closer spacings may show bigger differences between the schemes. In the remainder of this section only results using the original kinetic energy average are shown.

Figure 7 shows contours of absolute Mach number through the turbine at midspan. The absolute reference frame gives an unusual contour pattern in the rotors, but serves to show continuity at the averaging planes between the blade rows. Since the characteristic boundary conditions allow circumferential variations in the flow around some mean, Mach contours can be seen crossing the inlet and exit boundaries in several locations while the average Mach numbers are continuous across the interface. The contours also show the extremely thin blade boundary layers and wakes.

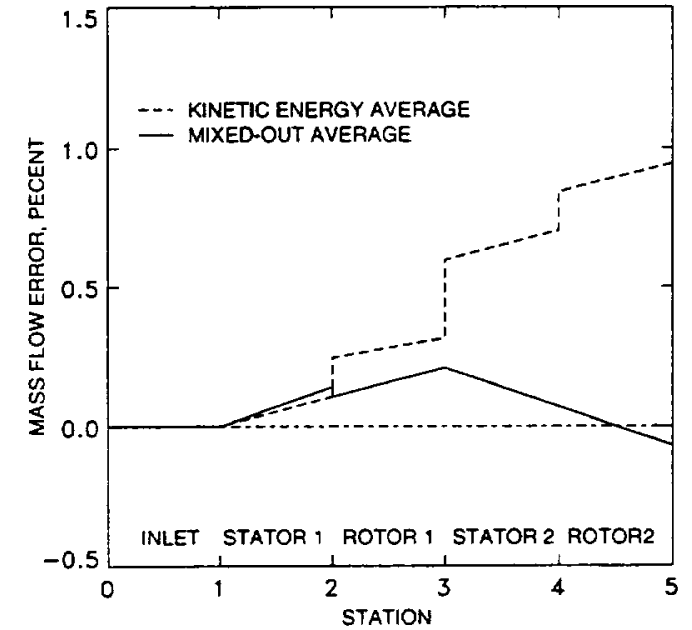

Figure 6 - Error in calculated mass flow at computational boundaries

Spanwise distributions of circumferentially-averaged total pressure ratio $\bar{P}_{0} / P_{0 \text { in }}$ are shown in figure 8 . The inlet profile shows the thin endwall boundary layers that were specified. The first stator generates about one percent loss in total pressure. The first rotor extracts work from the flow and drops the pressure ratio to about 0.815 , except near the tip where the clearance gap decreases the efficiency, leaving the pressure ratio slightly higher. The second stage performs like the first, giving an overall pressure ratio of about 0.67 .

Figure 9 compares computed and measured static pressures at various locations through the turbine. Computed stator surface pressures at midspan are compared to measured pressures from [26] (small circles). The computations agree very well with the data, except for small discrepancies on the uncovered portion of the suction surfaces. Computed pressures between the blade rows are shown along arbitrary grid lines at midspan. Since the characteristic boundary conditions allow circumferentially nonuniform pressure, the average (squares) and range (plus symbols) are shown at the interfaces. Endwall pressure measurements that have been averaged between the hub and tip are shown by large circles. Note that the measured exit static pressure ratio of 0.65 was set as the exit boundary condition for the computations. The agreement between the computed and measured average pressures between the blade rows is very good.

Figures $10-12$ show comparisons between computed and measured surface Stanton numbers at midspan plotted against unwrapped surface distance. The 


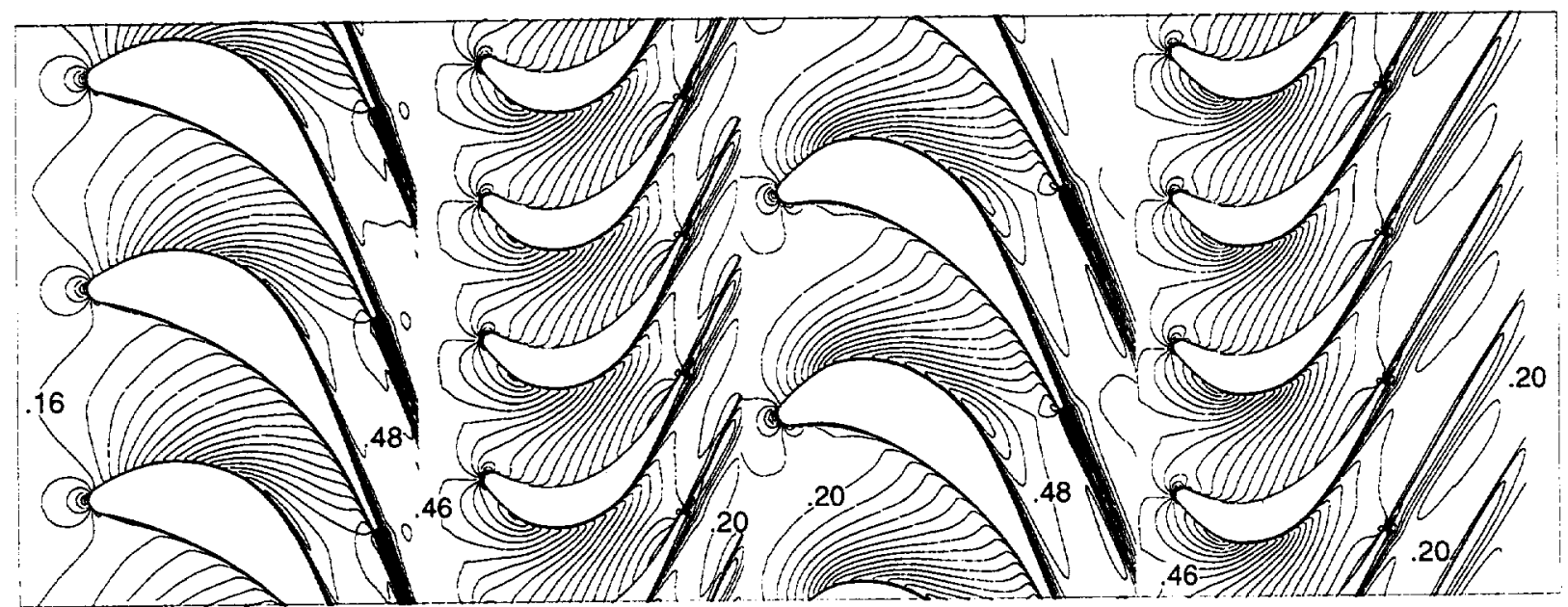

Figure 7 - Computed Mach number contours at mid span in the SSME turbine

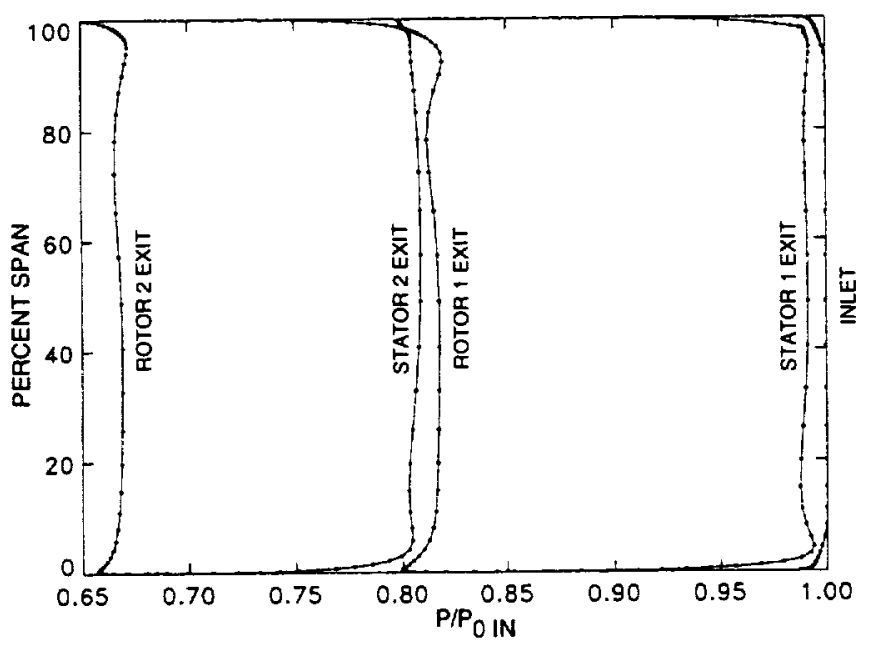

Figure 8-Computed spanwise distributions of total pressure in the SSME turbine

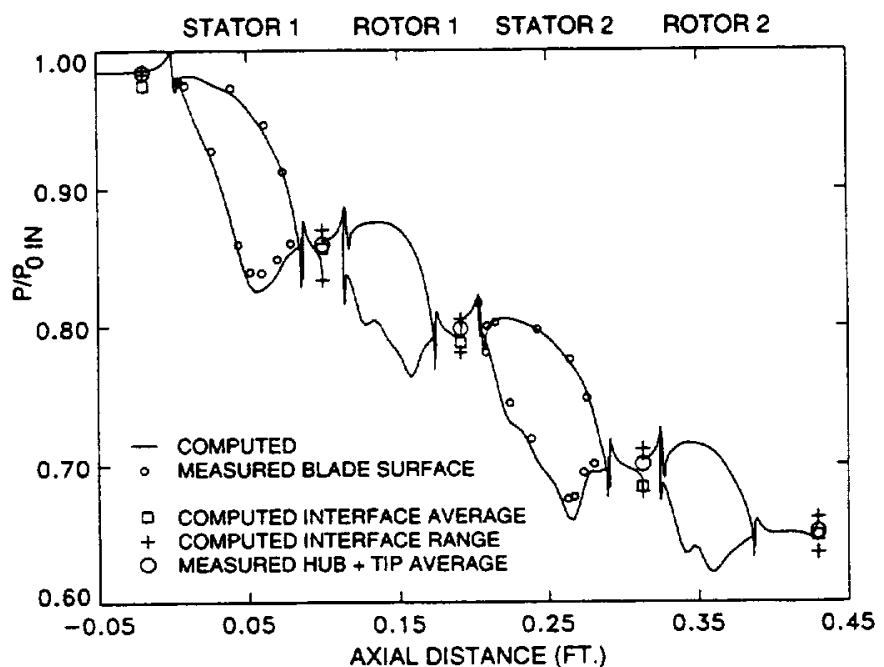

Figure 9 -Comparison of computed and measured static pressures in the SSME turbine 
pressure surface is denoted by negative distance. The Stanton number is defined by:

$$
\mathrm{S}_{t}=\frac{-\left.k \frac{\partial T}{\partial n}\right|_{w}}{\frac{\dot{m}}{A} C_{p}\left(T_{0}-T_{w}\right)}
$$

where $k$ is the gas conductivity, $(\partial T / \partial n)_{w}$ is the normal temperature gradient at the wall, $m / A$ is the mass flow per unit area, $C_{p}$ is the specific heat at constant pressure, $T_{0}$ is the inlet total temperature, and $T_{w}=0.7 T_{0}$ is the wall temperature.

Figure 10 shows Stanton numbers on stator 1 . The calculations match the high measured Stanton numbers near the stagnation point, but miss low values between $\pm(10$ to 20$)$ percent chord. The low Stanton numbers in this region probably indicate laminar or transitional boundary layers due to the low Reynolds number of this particular test [2]. The computations were run assuming fully-turbulent flow, which accounts for the discrepancies in heat transfer. Downstream of the transition region the computations are in better agreement with the data.

Subsequent blade rows experience unsteady perturbations from upstream wakes, which should shorten the transitional region. Figure 11 shows Stanton numbers on rotor 1 . The measurements show very high heat transfer at the leading edge which is almost predicted by the computations. On the suction surface the measurements show perhaps a small transitional region followed by fully-turbulent flow. The computations miss the transitional region but show excellent agreement in the turbulent region. Although the pressure surface was probably fully turbulent, the computed heat transfer is somewhat low.

Figure 12 shows Stanton numbers on stator 2. No transitional regions are evident in the data. The computed Stanton numbers show excellent agreement on the pressure surface but are somewhat high on the suction surface. No experimental data was taken on rotor 2 .

Overall it is felt that the computed Stanton numbers agree very well with the measurements. The results point out the need for reasonable transition models for multistage machines. With algebraic turbulence models it may be sufficient to model transition on the first blade row and leave subsequent rows fully turbulent. With multi-equation models the increased turbulent kinetic energy downstream of the first blade row may trigger early transition in later blade rows.

\section{Concluding Remarks}

A three-dimensional multiblock analysis code for turbomachinery was modified to allow analysis of multistage turbomachines. The SWIFT code was described briefly. The code can combine a limited selection of grid block types to simulate a wide range of turbomachinery problems. It uses an explicit finite-difference scheme to solve the thin-layer Navier-Stokes equations with the Baldwin-Lomax or Cebeci-Smith turbulence models. A spatially-varying time step, implicit residual smoothing, and preconditioning can be used to accelerate the convergence to a steady solution.

A steady averaging-plane method was used for multistage problems. Characteristic boundary conditions written in terms of linear perturbations about the average flow from the neighboring blade row were used to exchange information between the blade rows. The characteristic boundary conditions and the averagingplane implementation were described in detail. Two approaches for averaging the flow properties were also described.

A two-stage fuel turbine used on the space shuttle main engines was analyzed. Computed results were compared with experimental data from two independent tests. Surface pressure distributions on the stators and endwalls agreed very well with the experimental data except for slight discrepancies on the uncovered portion of the stator suction surfaces. Blade-surface distributions of heat transfer coefficient on the first three blade rows all compared very well with experimental data except in regions where transition was likely to be most important. Spanwise distributions of total pressure were shown but no data were available for comparison. Thus the ability of the method to predict overall performance of multistage turbomachines remains to be demonstrated.

Several conclusions regarding the characteristic boundary conditions and the averaging-plane method were reached:

1 The use of characteristic boundary conditions ensures that information propagates correctly between blade rows. Although the boundary conditions are nonreflecting, they did not change the convergence behavior of the code.

2 The linear formulation of the boundary conditions is easy to implement and behaves well numerically.

3 The use of perturbations about the average flow allows close spacing between the blade rows without forcing the flow to be axisymmetric. This property overcomes a main limitation of other averagingplane codes. 


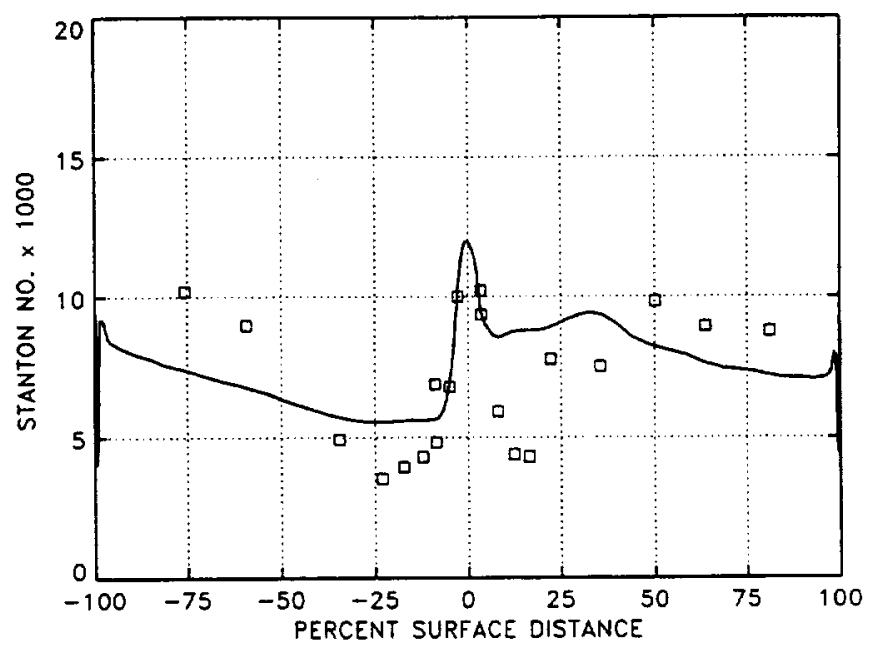

Figure 10 - Comparison of computed and measured Stanton numbers on stator 1

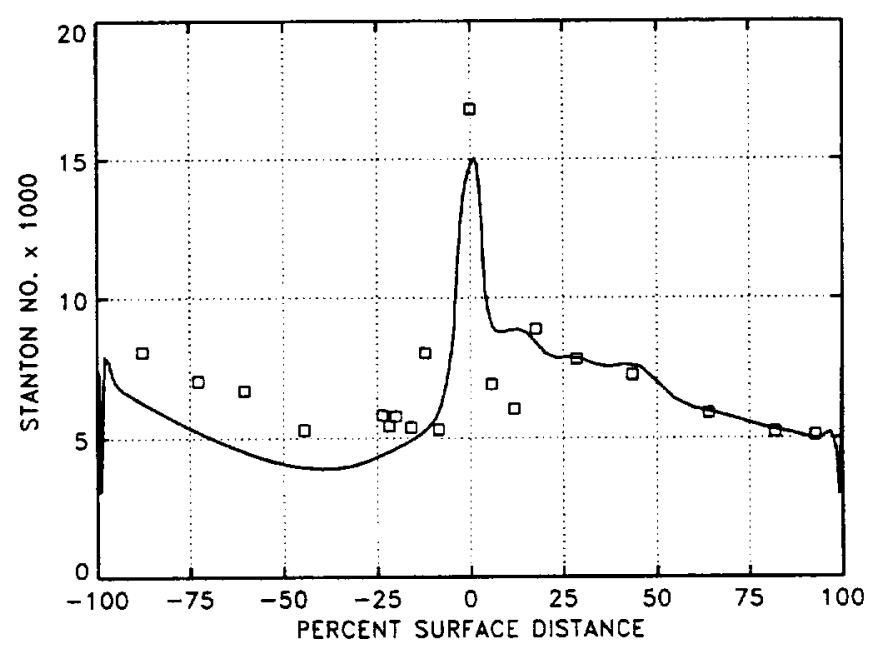

Figure 11 - Comparison of computed and measured Stanton numbers on rotor 1

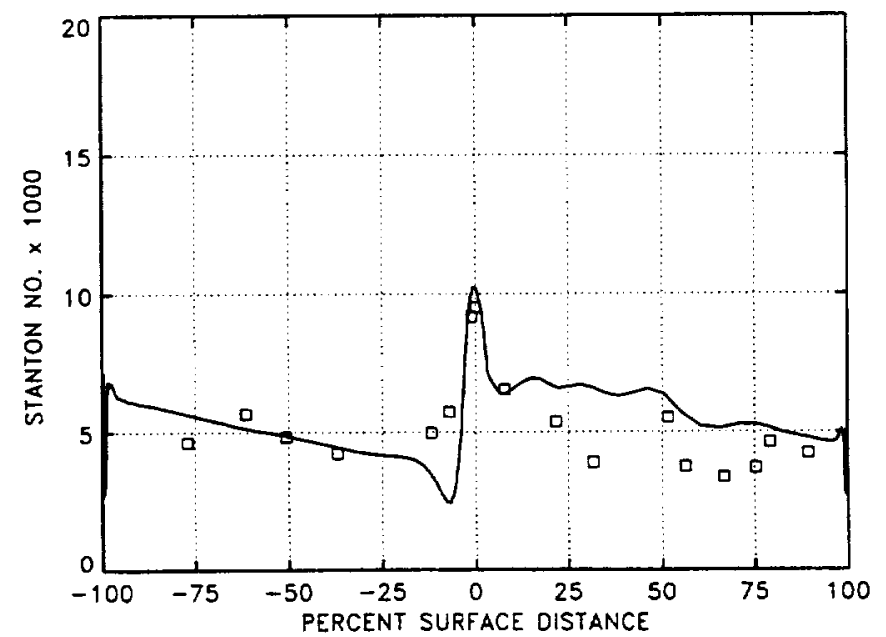

Figure 12 - Comparison of computed and measured Stanton numbers on stator 2 
4 The original boundary conditions exhibited small pressure perturbations where viscous wakes crossed the exit boundary. A modification to the linearization reduced this problem at exit boundaries, but the modification has not as yet been applied at averaging planes.

5 The well-known mixed-out average that represents the flow far downstream, and a new kinetic energy average that represents the local flow were used with the averaging-plane method. The mixed-out average had better conservation properties than the kinetic energy average, but no other significant differences were seen between the solutions in the low-speed case considered here. Larger differences may be expected at higher speeds or with closer blade spacings.

6 The addition of averaging-plane capability allows the SWIFT code to be used to analyze multistage turbomachinery efficiently. The method gives consistent spanwise solutions between blade rows that are difficult to obtain with successive analysis of isolated blade rows.

7 The averaging-plane method ignores physical processes such as wake mixing and migration, acoustic interaction, and other unsteady effects that may be important in real turbomachinery. The relative importance of these processes is unknown, and is likely to be highly case dependent.

\section{References}

1. Wyss, M. L., Chima, R. V., and Tweedt, D. L., "Averaging Techniques for Steady and Unsteady Calculations of a Transonic Fan Stage," AIAA Paper 93-3065, July 1993. Also NASA TM106231.

2. Boyle, R. J., and Giel, P. W., "Three-Dimensional Navier-Stokes Heat Transfer Predictions for Turbine Blade Rows," J. Propulsion and Power, Vol. 11, No. 6, Nov.-Dec. 1995, pp. 1179-1186.

3. Denton, J. D., "The Calculation of Three Dimensional Viscous Flow Through Multistage Turbomachines," ASME Paper 90-GT-19, June 1990.

4. Dawes, W. N., "Towards Improved Throughflow Capability: The Use of 3D Viscous Flow Solvers in a Multistage Environment," J. Turbomachinery, Vol. 114, pp. 8-17, 1992.
5. Arnone, A. and Benvenuti, E., "Three-Dimensional Navier-Stokes Analysis of a Two-Stage Gas Turbine," ASME Paper 94-GT-88, June 1994.

6. Hall, E. J., "Aerodynamic Modeling of Multistage Compressor Flowfields - Part 1: Analysis of Rotor/Stator/Rotor Aerodynamic Interaction," ASME Paper 97-GT-344, June 1997.

7. Hall, E. J., "Aerodynamic Modeling of Multistage Compressor Flowfields - Part 2: Modeling Deterministic Stresses," ASME Paper 97-GT345, June 1997.

8. Ni, R.-H., "Prediction of 3D Multi-stage Turbine Flow Field Using a Multiple-Grid Euler Solver," AIAA Paper 89-0203, Jan. 1989.

9. Saxer, A. P., and Giles, M. B., "Predictions of Three-Dimensional Steady and Unsteady Inviscid Transonic Stator/Rotor Interaction With Inlet Radial Temperature Nonuniformity," J. Turbomachinery, Vol. 116, July 1994, pp. 347-357.

10. Adamczyk, J. J., "Model Equation for Simulating Flows in Multistage Turbomachinery," ASME Paper 85-GT-226, Mar. 1985. Also NASA TM86869.

11. Adamczyk, J. J., Mulac, R. A., and Celestina, M. L., "A Model for Closing the Inviscid Form of the Average-Passage Equation System, "ASME Paper 86-GT-227, June 1986. Also NASA TM87199.

12. Adamczyk, J. J., Celestina, M. L., and Chen, J. P., "Wake-Induced Unsteady Flows: Their Impact on Rotor Performance and Wake Rectification," ASME Paper 94-GT-219, June 1994.

13. Rhie, C., Gleixner, A. J., Spear, D. A., Fischberg, C. J., and Zacharias, R. M., "Development and Application of a Multistage Navier-Stokes Solver, Part I: Multistage Modeling Using BodyForces and Deterministic Stresses," ASME Paper 95-GT-342, June 1995.

14. LeJambre, C. R., Zacharias, R. M., Biederman, B. P., Gleixner, A. J., and Yetka, C. J., "Development and Application of a Multistage NavierStokes Flow Solver, Part II: Application to a High Pressure Compressor Design," ASME Paper 95GT-343, June 1995. 
15. Rai, M. M., "Unsteady Three-Dimensional Navier-Stokes Simulations of Turbine Rotor-Stator Interaction," J. Propulsion and Power, Vol. 5, No. 3, May-June 1989, pp. 307-319.

16. Arnone, A. and Pacciani, R., "IGV-Rotor Interaction in a Transonic Compressor Using the NavierStokes Equations," ASME Paper 96-GT-141, June 1996.

17. Giles, Michael B., "Nonreflecting Boundary Conditions for Euler Equation Calculations," AIAA Journal, Vol. 28, No. 12, Dec. 1990, pp. 2050-2058.

18. Chima, Rodrick V., "Calculation of Tip Clearance Effects in a Transonic Compressor Rotor," ASME Paper 96-GT-114, June 1996. Also NASA TM-107216.

19. Chima, R. V., and Yokota, J. W., "Numerical Analysis of Three-Dimensional Viscous Flows in Turbomachinery," AIAA J., Vol. 28, No. 5, May 1990, pp. 798-806.

20. Chima, R. V., "Viscous Three-Dimensional Calculations of Transonic Fan Performance," in CFD Techniques for Propulsion Applications, AGARD Conference Proceedings No. CP-510, AGARD, Neuilly-Sur-Seine, France, Feb. 1992, pp 21-1 to 21-19. Also NASA TM-103800.

21. Chima, R. V., Giel, P. W., and Boyle, R. J., "An Algebraic Turbulence Model for Three-Dimensional Viscous Flows," AIAA Paper 93-0083, Jan. 1993. Also NASA TM-105931.

22. Jameson, A., Schmidt, W., and Turkel, E., "Numerical Solutions of the Euler Equations by Finite Volume Methods Using Runge-Kutta Time-Stepping Schemes," AIAA Paper 81-1259, June 1981.

23. Kunz, R. F., and Lakshminarayana, B. "Explicit Navier-Stokes Computation of Cascade Flows Using the k- $\varepsilon$ Turbulence Model," AIAA J., Vol. 30, No. 1, Jan. 1992, pp. 13-22.

24. Tweedt, D. L., Chima, R. V., and Turkel, E. "Preconditioning for Numerical Simulation of Low Mach Number Three-Dimensional Viscous Turbomachinery Flows," AIAA Paper 97-1828, June, 1997.
25. Dunn, M. G., Kim, J., Civinskas, K. C., and Boyle, R. J., "Time-Averaged Heat Transfer and Pressure Measurements and Comparison With Prediction for a Two-Stage Turbine," J. Turbomachinery, Vol. 116, Jan. 1994, pp. 14-22.

26. Hudson, S. T., Gaddis, S. W., Johnson, P. D., and Boynton, J. L., "Cold Flow Testing of the Space Shuttle Main Engine High Pressure Fuel Turbine," AIAA Paper 91-2503, June 1991. 
. 


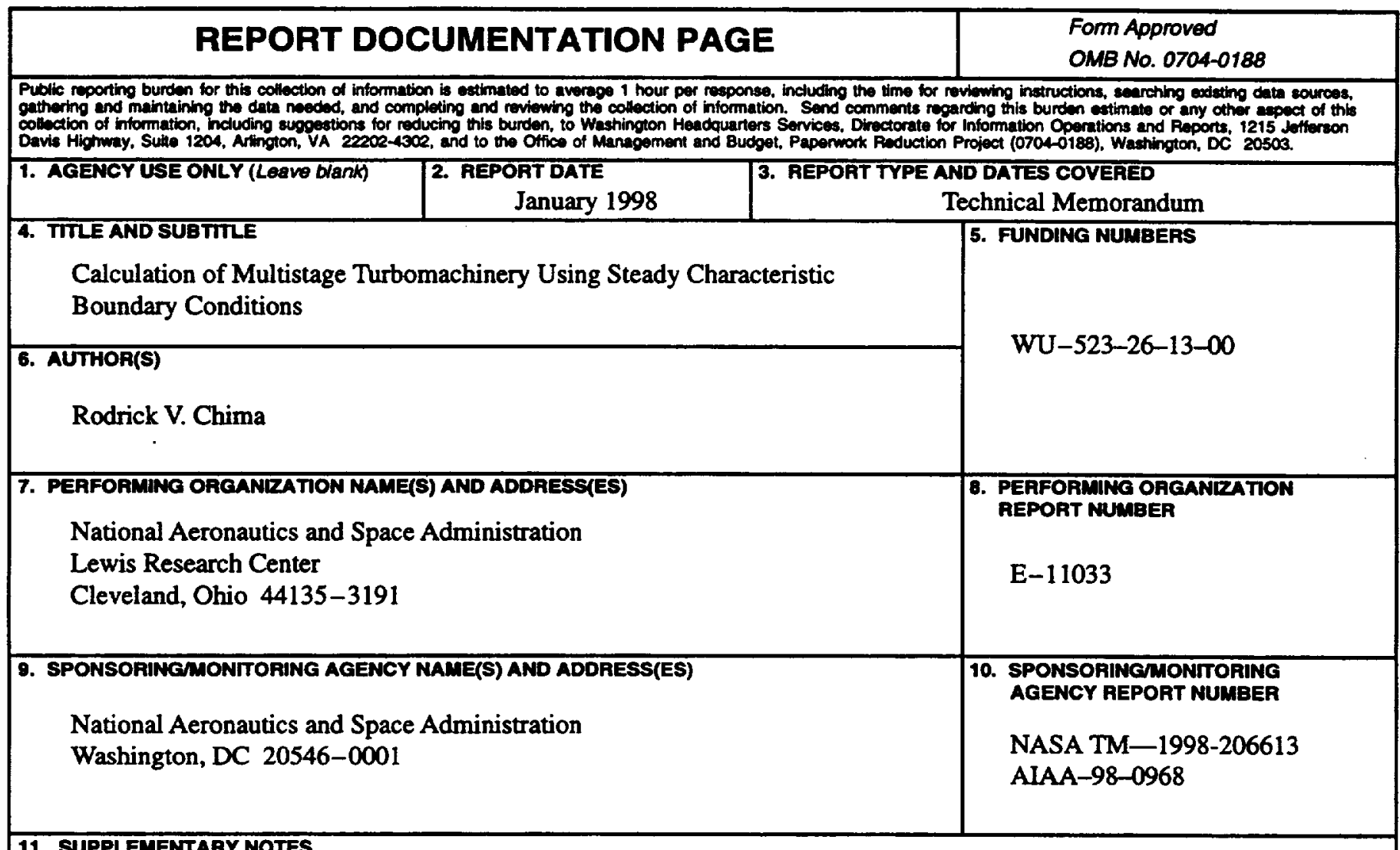

Prepared for the 36th Aerospace Sciences Meeting \& Exhibit sponsored by the American Institute of Aeronautics and Astronautics, Reno, Nevada, January 12-15, 1998. Responsible person, Rodrick V. Chima, organization code 5810, (216) 433-5919.

12a. DISTRIBUTIONAVALABILTY STATEMENT

12b. DISTAIBUTION CODE

Unclassified - Unlimited

Subject Category: 07

Distribution: Nonstandard

This publication is available from the NASA Center for AeroSpace Information, (301) 621-0390.

13. ABSTRACT (Maximum 200 mords)

A multiblock Navier-Stokes analysis code for turbomachinery has been modified to allow analysis of multistage turbomachines. A steady averaging-plane approach was used to pass information between blade rows. Characteristic boundary conditions written in terms of perturbations about the mean flow from the neighboring blade row were used to allow close spacing between the blade rows without forcing the flow to be axisymmetric. In this report the multiblock code is described briefly and the characteristic boundary conditions and the averaging-plane implementation are described in detail. Two approaches for averaging the flow properties are also described. A two-dimensional turbine stator case was used to compare the characteristic boundary conditions with standard axisymmetric boundary conditions. Differences were apparent but small in this low-speed case. The two-stage fuel turbine used on the space shuttle main engines was then analyzed using a three-dimensional averaging-plane approach. Computed surface pressure distributions on the stator blades and endwalls and computed distributions of blade surface heat transfer coefficient on three blades showed very good agreement with experimental data from two tests.

\begin{tabular}{|c|c|c|}
\hline \multicolumn{3}{|c|}{$\begin{array}{l}\text { 14. SUBJECT TERMS } \\
\text { Multistage turbomachinery; Characteristic boundary conditions; Computational fluid } \\
\text { dynamics; Space shuttle main engine; Turbines; Heat transfer }\end{array}$} \\
\hline $\begin{array}{l}\text { 17. SECUAITY CLASSIFICATION } \\
\text { OF REPORT } \\
\text { Unclassified }\end{array}$ & $\begin{array}{l}\text { 18. SECURITY CLASSIFICATION } \\
\text { OF THIS PAGE } \\
\text { Unclassified }\end{array}$ & $\begin{array}{l}\text { 19. SECURTY CLASSIFICATION } \\
\text { OF ABSTRACT } \\
\text { Unclassified }\end{array}$ \\
\hline
\end{tabular}

15. NUNBEA OF PAGES 20

16. PRICE CODE

$\mathrm{A} 03$

20. UMITATION OF ABSTRACT

Standard Form 298 (Rev. 2-89)

Prescribed by ANSI Sid. Z39-18 298-102 